zen etwas schwächer. Alle am Leben gebliebenen Versuchspfropfungen (19) blühten, alle Kontrollpfropfungen (8) blieben bis Anfang Januar 1954 vegetativ. Selbstverständlich wurde seit der Zeit natürlichen Kurztags für ausreichende künstliche Tagesverlängerung durch „Osram Nitra“ und „Fluoreszenz HNG-Lampen“ gesorgt. Abb. 2 zeigt ausgewählte Beispiele.

Das Ergebnis unserer Versuche ist also, daß Kalanchoё ein für Transplantationsversuche besonders geeignetes Objekt ist. Auch einzelne Blätter lassen sich leicht transplantieren, wie noch ergänzend erwähnt werden soll. Mit dem viel härteren Sproßgewebe von Sedum kamtschaticum läßt sich nicht

1 H. A. A 11 a r d, J. agric. Res. 74, 15-31 [1947].

2 M. C. Č a j l a c h j a n, C. R. [Doklady] Acad. Sci. URSS 13 N.S., $79-83$ [1936].

3 D. v. D e n f f e r, Jahrb. wiss. Bot. 89, 543-573 [1941].

4 R. H a r d e r, Symposia Soc. exp. Biol. Cambr. II Growth [1948].

5 R. H a rd e r u. G. G ü m m e r, Jahrb. wiss. Bot. 91, 359-380 [1944]. ganz so leicht hantieren. Ein Nachteil der Kalanchö̈ ist die langsame Reaktion auf die Pfropfung. Die Sukkulenz des Blattgewebes bietet für die experimentelle Behandlung mancher Frage aber vielleicht so viele Vorteile, daß man auf die Transplantationstechnik bei der Behandlung von Fragen der Physiologie der Blütenbildung auch bei Kalanchoë in $\mathrm{Zu}$ kunft nicht verzichten sollte. Die Sukkulenz bringt aber eine Gefahr mit sich, an der frühere Transplantationsversuche vielleicht gescheitert sind: Man bemerkt das Welken der Pfropfreiser nicht rechtzeitig, wenn man die Pfropfungen in den ersten Tagen nicht beobachtet und nicht für ausreichende Luftfeuchtigkeit zur Verhinderung des Welkens sorgt.

6 J. Kuijper u. L. K. Wi ersum, Proc. Kon. Akad. Wetensch. Amsterdam, 39, 1-10 [1936].

7 A. L a n g, Ann. Rev. Plant. Physiol. III [1952].

8 G. M e l ch ers , Biol. Zbl. 56, 567-570 [1936].

9 B. S. Moschkov, C. R. [Doklady] Acad. Sci. URSS 31, 161-162 [1941].

10 R. H. Roberts u. B. E. Struckmeyer, Lotsya, Vol. 1 (Vernalisation and Photoperiodism) Waltham, Mass. U.S.A. 1948.

\title{
Vergleichende Atmungsmessungen an Sommer- und Winterannuellen
}

\author{
Untersuchungen an Caryopsen und Embryonen von Secale cereale \\ und an Samen von Arabidopsis thaliana \\ Von Klaus Napp-ZinN \\ Aus dem Max-Planck-Institut für Biologie, Abt. Melchers, Tübingen \\ (Z. Naturforschg. 9 b, 218-229 [1954]; eingegangen am 27. Januar 1954)
}

\begin{abstract}
Die Atmungsintensität der Caryopsen bzw. der Samen sommerannueller Rassen von Secale cereale und Arabidopsis thaliana lag in kurzfristigen Versuchen bei allen angewandten Temperaturen $\left(+2^{\circ}\right.$ bis $\left.40^{\circ}\right)$ höher als bei den entsprechenden winterannuellen Formen. Das gleiche gilt auch für Roggen-Caryopsen im 5 -wöchigen Vernalisations-Versuch bei $2^{\circ}$, bei dessen Ende die Atmung des Sommerroggens noch weiter stieg, die des Winterroggens hingegen ihr Maximum erreicht hatte. Bei isolierten Embryonen des Winterroggens stieg die Atmungsintensität im 4-wöchigen Vernalisations-Versuch bei $2^{\circ}$ anfangs langsamer, später schneller als beim Sommerroggen; das Maximum trat bei beiden etwa am 13. Versuchstag ein und lag beim Winterroggen erheblich höher als beim Sommerroggen.

Der $R Q$ lag bei Embryonen und Caryopsen des Sommerroggens allgemein höher als die entsprechenden Werte des Winterroggens. Für kurzfristige Versuche ist dieser Unterschied vorzüglich statistisch gesichert und für langfristige Versuche (von denen bei Caryopsen und Embryonen des Sommer- und des Winterroggens nur je 2 Parallelen durchgeführt wurden) wahrscheinlich. Der $R Q$ liegt beim Roggen bei $20^{\circ}$ um 1, bei tieferen Temperaturen tiefer und bei höheren höher.

Bei Arabidopsis thaliana hingegen lag der $R Q$ der Samen der sommerannuellen Rasse $\mathrm{Li}_{\text {, }}$ durchweg tiefer als der der winterannuellen Rasse St, hatte aber sein Maximum (etwa 0,7) im Bereich zwischen 10 und $20^{\circ}$ und sank sowohl bei (vernalisierenden) tieferen als auch bei (devernalisierenden) höheren Temperaturen auf tiefere Werte.

Die Ergebnisse werden, anknüpfend an frühere Befunde von Gregory und Purvis, im Hinblick auf etwaige Zusammenhänge zwischen Atmung und Vernalisation diskutiert.
\end{abstract}


Ü: ber die dem Vernalisations-Effekt zugrunde liegenden physiologisch-chemischen Vorgänge ist nur sehr wenig bekannt. Richter, R a n c a n und P e k k a r ${ }^{1}$ stellten im Verlauf der Kältebehandlung zweier Winterweizen-Rassen Änderungen der Aktivität einiger Fermente fest, und D u pér o n², Da $\mathrm{vid}^{3}$ und Séchet $\mathrm{t}^{4}$ fanden Verschiebungen im Reservestoffgehalt von Wintergetreiden und Radieschen bei Vernalisation im Frucht- bzw. Samenstadium, ohne daß die betreffenden Befunde Aussagen über den eigentlichen Vernalisations-Vorgang und die dabei unmittelbar beteiligten Substanzen gestatteten, zumal Vergleiche mit den entsprechenden sommerannuellen Formen vielfach fehlten. Die Vorstellungen über die inneren Vorgänge bei Vernalisation und Devernalisation (Lang und $\mathrm{Melchers^{5 }}$, Melchers $^{6}$, Purvis und Gregory ${ }^{7}, \quad \mathrm{Napp}$ $\mathrm{Z}$ in ${ }^{8}$ ) sind daher noch rein formal und lassen die Frage nach der chemischen Natur dieser Prozesse offen.

Wohl aber hatten frühere Versuche von Gregory und Purvi s ${ }^{9}$ gezeigt, daß der Vernalisations-Effekt tiefer Temperaturen bei Embryonen von Wintergetreiden, insbesondere des Winterroggens, nur in Anwesenheit von $\mathrm{O}_{2}$ eintritt, daß 1-3-wöchige Aufbewahrung gequollener Caryopsen in $\left(\mathrm{O}_{2}\right.$-freier $)$ Stickstoffatmosphäre auch bei Sommerroggen devernalisierend wirkt und daß bei isolierten Embryonen, zumal nach mehrtägiger Vorquellung bei $20^{\circ}$ (d. h. nach Veratmung der vorhandenen Kohlenhydrate), die Zugabe von Glucose zum Nährmedium das Eintreten des Vernalisations-Effektes wesentlich beschleunigt, wenn nicht überhaupt erst ermöglicht. (P u r vis $\left.{ }^{10,11}\right)$. - Darüber hinaus erkannten Chouard und $\mathrm{Poignant}{ }^{12}$ an Winterweizen die vernalisationshemmende Wirkung einiger Atmungsgifte.

Diese Befunde veranlaßten uns, nach etwaigen Zusammenhängen zwischen dem Atmungsstoffwech-

1 A. A. Richter, V. Rancan u. M. Pekka, C. R. [Doklady] Acad. Sci. URSS 1933 (2), 72-75.

2 R. D u p é r o n, Rev. gén. Bot. 53, 525-557 [1946]; C. R. hebd. Séances Acad. Sci. 228, 192-194 [1949].

3 R. D a vid, C. R. hebd. Séances Acad. Sci. 228, 1242-1243 [1949]; Ann. Biol. 26, 413-445 [1950].

4 R. D a vid u. J. S é c h e t, C. R. hebd. Séances Acad. Sci. 227, 537-539 [1948].

5 A. Lang u. G. Melchers, Z. Naturforschg. 2b, $444-449$ [1947].

6 G. Melchers, Ist. Lomb. Sci. e Lett., Rendiconti, Cl. Sci. 83, 1-29 [1950]; The physiology of flowerinitiation. 33 pp. Als Mskr. vervielfältigt. Göttingen 1952.

7 O. N. Purvis u. F. G. Gregory, Ann. Botany, N.S. 16, 1-21 [1952]. sel einerseits, dem Kältebedürfnis winterannueller Pflanzen sowie dem Fehlen des Kältebedürfnisses bei sommerannuellen Pflanzen andererseits zu suchen.

\section{Untersuchungen am Roggen}

Die Messungen wurden zunächst ausschließlich an Caryopsen und isolierten Embryonen des Sommerund des Winterroggens ausgeführt (Material: Petkuser Normalstroh-Winterroggen, Hochzucht, bzw. Petkuser Sommerroggen, Hochzucht, der Fa. F. von Lochow-Petkus GmbH., Bergen, Kreis Celle, Ernten 1950 und 1951)*. Die Verwendung von Roggen empfahl sich natürlich im Hinblick auf die Anknüpfung an die erwähnten Arbeiten von G r e g o r y und Purvis, ganz abgesehen davon, daß Wintergetreide als Früchte oder Embryonen vernalisiert werden können und diese als Versuchsobjekte für diese Fragestellung viel handlicher sind als etwa die Rosetten zweijähriger Pflanzen.

\section{A. Kurzfristige Versuche 1. Ganze Caryopsen}

a) Methode

Zunächst wurden kurzfristige Untersuchungen an ganzen Caryopsen vorgenommen. Diese wurden in Mengen von etwa je $10 \mathrm{~g}$ in einer Petrischale etwa $2 \mathrm{Min}$. lang mit 95-proz. Alkohol oberflächlich sterilisiert, sodann 3-mal mit dest. Wasser ausgewaschen, anschließend zwecks weiterer Sterilisation $1 \frac{1}{2} 2$ Stdn. lang in einer 10-proz. Chlorkalkaufschwemmung gehalten, wiederum 3-mal mit dest. Wasser gespült und schließlich über Filtrierpapier $23 \mathrm{Stdn}$. lang in $15 \mathrm{~cm}^{3}$ dest. Wassers im Dunkeln (im Brutschrank oder in der physiologischen Dunkelkammer) bei $23 \pm 1 / 2^{\circ} \mathrm{C}$ vorgequollen. Da jedes Desinfektionsmittel bekanntlich auch den Stoffwechsel der zu sterilisierenden Pflanzenorgane mehr oder weniger stark beeinflußt (vgl. besonders Pringsheim ${ }^{13}$ und Denny 14), wurde auf die Gleichmäßigkeit dieser Vorbehandlung größter Wert gelegt. - Nun wurden nach abermals 3-maliger

8 K. N a p p-Zinn, Ber. dtsch. bot. Ges. 66, 362-367 [1953].

9 F. G. Gre gory u. O. N. Purvis, Ann. Botany, N.S. 2, 753-764 [1938].

10 O. N. Purvis, Ann. Botany, N.S. 8, 285-314 [1944].

11 O. N. Purvis, Ann. Botany, N.S. 11, 269-283 [1947].

12 P. Chou ard u. P. Poignant, C. R. hebd. Séances Acad. Sci. 232, 103-105 [1951].

13 E. G. Pring s h e i m, Angew. Bot. 10, 208-279 [1928].

14 F. E. Denny, Contr. Boyce Thompson Inst. 15, 211-227 [1948].

* Ich danke der Firma Lochow für die bereitwillige Überlassung des Saatguts. 


\begin{tabular}{|c|c|c|c|c|c|c|}
\hline \multicolumn{2}{|c|}{ Temperatur } & $2^{\circ} \mathrm{C}$ & $10^{\circ} \mathrm{C}$ & $20^{\circ} \mathrm{C}$ & $30^{\circ} \mathrm{C}$ & $40^{\circ} \mathrm{C}$ \\
\hline \multirow{2}{*}{$\begin{array}{c}\mathrm{CO}_{2^{-}} \\
\text {Abgabe }\end{array}$} & $\begin{array}{l}\mathrm{S} \\
\mathrm{W}\end{array}$ & $\begin{array}{ll}14,22 & (4) \\
12,23 & (3)\end{array}$ & $\begin{array}{ll}34,0 & (5) \\
30,74 & (5)\end{array}$ & $\begin{array}{ll}86,0 & (4) \\
71,8 & (2)\end{array}$ & $\begin{array}{ll}139,3 & (2) \\
136,9 & (2)\end{array}$ & $\begin{array}{ll}211 & (1) \\
144,7 & (1)\end{array}$ \\
\hline & $P$ & 0,0026 & 0,018 & 0,0003 & $>0,3$ & - \\
\hline \multirow{2}{*}{$\begin{array}{c}\mathrm{O}_{2^{-}}^{-} \\
\text {Verbrauch }\end{array}$} & $\begin{array}{l}\mathrm{S} \\
\mathrm{W}\end{array}$ & $\begin{array}{l}24,44(4) \\
22,155(3)\end{array}$ & $\begin{array}{ll}43,66 & (5) \\
42,02 & (5)\end{array}$ & $\begin{array}{ll}87,8 & (4) \\
72,9 & (2)\end{array}$ & $\begin{array}{ll}107,4 & (2) \\
116,4 & (2)\end{array}$ & $\begin{array}{ll}152,7 & (1) \\
108,3 & (1)\end{array}$ \\
\hline & $P$ & 0,00037 & 0,23 & 0,00033 & $>0,3$ & - \\
\hline \multirow{2}{*}{$R Q$} & $\begin{array}{l}\mathrm{S} \\
\mathrm{W}\end{array}$ & $\begin{array}{l}0,582(4) \\
0,552(3)\end{array}$ & $\begin{array}{ll}0,778 & (5) \\
0,732 & (5)\end{array}$ & $\begin{array}{l}0,980(4) \\
0,984(2)\end{array}$ & $\begin{array}{l}1,300(2) \\
1,180(2)\end{array}$ & $\begin{array}{l}1,371(1) \\
1,334(1)\end{array}$ \\
\hline & $P$ & 0,064 & 0,00036 & $>0,3$ & 0,028 & - \\
\hline
\end{tabular}

Tab. 1. Statistische Sicherung der Unterschiede in der $\mathrm{CO}_{2}$-Abgabe, dem $\mathrm{O}_{2}$-Verbrauch (beide in $\mathrm{mm}^{3}$ pro Stde. und g Trockengewicht) und dem $R Q$ von Caryopsen des Sommer- (S) und des Winterroggens (W) nach 23-stdg. Quellung bei $23^{\circ} \mathrm{C}$. $P$-Werte nach $\mathrm{P}$ ä $\mathrm{t} \mathrm{a} \mathrm{u}{ }_{1}{ }^{23}$ berechnet. In Klammern jeweils die Anzahl der Parallelmessungen.

Waschung mit dest. Wasser je 20 Caryopsen (auf deren äußerliche Gleichartigkeit stets geachtet wurde) auf Filtrierpapier flüchtig abgetrocknet und in die mit $2,4 \mathrm{~cm}^{3}$ dest. Wassers beschickten Reaktionsgefäße einer Warburg-Apparatur (Volumen etwa $18 \mathrm{~cm}^{3}$ ) gegeben. In Abweichung von Pringsheims ${ }^{15}$ Versuchsanordnung bevorzugten wir diese geringe Wassermenge an Stelle von Luft, um den Wassergehalt der Caryopsen und damit die Diffusionsmöglichkeiten für Gase einigermaßen konstant zu halten.

Auf die Nachteile der manometrischen Methode nach W a rburg ${ }^{16}$ für Atmungsmessungen an keimenden Samen haben vor allem Pringsheim ${ }^{15}$ und auch Ullrich und Ruhland ${ }^{17}$ hingewiesen; es handelt sich dabei in erster Linie um den geringen Rauminhalt der Reaktionsgefäße, ihre darauf beruhende geringe Aufnahmefähigkeit für Versuchsmaterial und die hierdurch bedingte größere Wahrscheinlichkeit zufälliger Ergebnisse sowie um die Aussichtslosigkeit, zwei sich respiratorisch völlig gleich verhaltende Samenproben zu finden. Diese Nachteile scheinen jedoch durch die Gewähr hoher Ablesegenauigkeit, durch ein Minimum an apparativen Schwierigkeiten und die Einfachheit der Berechnung der Ergebnisse weitgehend aufgewogen, obwohl sich die $\mathrm{Be}-$ nutzung verschiedener Proben für die $\mathrm{O}_{2}$ - und die $\mathrm{CO}_{2}$ Bestimmung nicht umgehen ließ ${ }^{*}$.

In den für erstere vorgesehenen Gefäßen wurde das freiwerdende $\mathrm{CO}_{2}$ in $1 \mathrm{~cm}^{3} n . \mathrm{KOH}$ absorbiert. Bei den übrigen Gefäßen wurde bei Versuchsbeginn oder bei

* Andererseits hätte die Benutzung verschiedener Methoden zur $\mathrm{CO}_{2}$ - und $\mathrm{O}_{2}$-Bestimmung leicht unvergleichbare Werte ergeben können, wie es bei den Versuchen von Gowdridge und Thoday 18 infolge des frühzeitigen Todes der von ihnen untersuchten Compositenblätter in dem zur $\mathrm{O}_{2}$-Verbrauchs-Bestimmung benutzten $\mathrm{B}$ a r c r of t-Respirometer in der Dix o n schen Modifikation anfangs der Fall war.

15 E. G. Pringsheim u. Mit a rbb., Planta 15 , 419-458 [1931]; Planta 19, 653-712 [1933].
Versuchsschluß $1 \mathrm{~cm}^{3} n$. $\mathrm{HCl}$ eingekippt und die alsdann im Verlauf 1 Stde. aus Gewebe und Medium austretende $\mathrm{CO}_{2}$-Menge ermittelt. Auf diese Weise wurde der Retention von $\mathrm{CO}_{2}$ Rechnung getragen, die $\mathrm{Pr}$ ing sh e i m ${ }^{15}$ als unvermeidliche Fehlerquelle betrachtet hatte und auf deren Bedeutung in neuerer Zeit wieder Denny ${ }^{19}$ und $\mathrm{Scott} 20$ hingewiesen haben (vgl. auch Dickens in B a mann und My rbäck21, S. 1006/1007). Die eigentliche Versuchsdauer betrug bei $2^{\circ} \mathrm{C} 16 \mathrm{Stdn}$., bei allen anderen Temperaturen $6 \mathrm{Stdn}$. Die Schüttelfrequenz der Manometer belief sich auf 60 Doppelschläge pro Minute.

Nach Versuchsschluß wurden die Caryopsen 4 Stdn. lang bei $105^{\circ} \mathrm{C}$ getrocknet und endlich gewogen, so daß die Resultate der Messungen auf das Trockengewicht bezogen werden konnten.

b) Ergebnis s e

Es versteht sich von selbst, daß für eine möglichst weitgehende statistische Sicherung der für $\mathrm{CO}_{2}$-Produktion, $\mathrm{O}_{2}$-Verbrauch und respiratorischen Quotienten $(R Q)$ ermittelten Werte mehrere Wiederholungen jedes Einzelversuchs (mit gleicher Temperatur und gleichem Objekt) erforderlich waren. Innerhalb weniger Tage pflegten diese stets ganz ähnliche Ergebnisse zu zeitigen; bei größeren Abständen zwischen den einzelnen Versuchen einer Serie traten dagegen oft

16 O. W a r burg, Biochem. Z. 100, 230 [1919].

17 H. Ullrich u. W. Ruhl and, Planta 5, 360-380 [1928].

18 B. M. Gowdridge u. D. Thoday, Ann. Botany, N.S. 16, 349-372 [1952].

19 F. E. D e n ny, Contr. Boyce Thompson Inst. 14, $257-276,315-322,383-396$ [1946/47].

20 J. K. S c ot t, Diss. Cambridge [England] 1949.

21 E. B a mann u. K. M y r bäck, Die Methoden der Fermentforschung 2. Leipzig 1941. 
erhebliche Abweichungen hinsichtlich Atmungsintensität und $R Q$ auf, die sich dann wiederum tagelang gut reproduzieren ließen. Diese unerwarteten und unwillkommenen Verschiebungen erfolgten bei Sommer- und Winterroggen stets in gleichem Sinne und legen die Annahme einer vielleicht jahreszeitlich bedingten Änderung des physiologischen Zustandes der Caryopsen nahe, da evtl. kleine Schwankungen der Versuchsbedingungen zur Erklärung dieser Unterschiede nicht ausreichen und auch - auf Grund von Vergleichen zwischen den an Caryopsen der Ernten 1950 und 1951 erhaltenen Ergebnissen - altersbedingte Änderungen, wie sie Roberts ${ }^{22}$ bei Triticum dicoccum gefunden hatte, nicht in Betracht kommen. Im folgenden werden daher in diesem $\mathrm{Ab}$ schnitt nur diejenigen Ergebnisse berücksichtigt, die in der Zeit vom 14. 2. bis 28. 3. 1952 an Material der Ernte 1951 gewonnen wurden.

Wie Abb. 1 zeigt, liegen die Werte für die $\mathrm{CO}_{2}$ Abgabe und den $\mathrm{O}_{2}$-Verbrauch des Sommerroggens bei den angewandten Temperaturen $\left(+2^{\circ}, 10^{\circ}, 20^{\circ}\right.$, $30^{\circ}$ und $40^{\circ} \mathrm{C}$ ) im allgemeinen höher als die entsprechenden Werte beim Winterroggen, was nicht erstaunlich ist, da der Sommerroggen auch bei der Keimung dem Winterroggen vorauseilt und Atmung und Keimung weithin parallel verlaufen. Die einzige Abweichung von dieser Regel $\left(\mathrm{O}_{2}\right.$-Verbrauch bei $\left.30^{\circ}\right)$ ist statistisch ungesichert (vgl. Tab. 1). - Das totale $P$, das nach $\mathrm{P}$ äta ${ }^{24}$ ermittelt wurde, liegt bei $\mathrm{CO}_{2}$-Abgabe und bei $\mathrm{O}_{2}$-Verbrauch (unter Zugrundelegung der bei $2-30^{\circ}$ erhaltenen Meßwerte) in der Größenordnung von 1,2 bzw. 2,5 $10^{-6}$.

Der $R Q$-Wert des Sommerroggens liegt über dem des Winterroggens. Auch hier ist die einzige Ausnahme $\left(20^{\circ}\right)$ statistisch nicht gesichert, während das totale $P$ etwa 0,00005 beträgt und gute Sicherung der Differenz bekundet. Die relativ hohen $R Q$-Werte bei $30^{\circ}$ und $40^{\circ}$ bringen die bei diesen Temperaturen trotz guter Aëration einsetzende Gärung zum Ausdruck, doch wurden die von Frietinger ${ }^{25}$ schon bei tieferer Temperatur erzielten hohen Werte (die gewiß auf mangelnde Belüftung zurückzuführen

* Schon M o is s o u 26 hatte - in späterem Gegensatz zu Bonnier und Mangin 27 - die Temperaturabhängigkeit des $R Q$ erkannt; vgl. auch $\mathrm{H}$ a rring t on 28 .

22 D. W. A. R o b e r t s, Canad. J. Botany 29, 10-25 [1951].

23 K. P ät a u , Biol. Zbl. 63, 152-168 [1943].

24 K. P ät a u , Z. Vererbungslehre 80, 558 - 564 [1942].

25 G. Frieting er, Flora 22, 167-201 [1927].

26 H. M o is s ou, Ann. Sci. natur., Bot., Sér. 6, 6, $292-339$ [1878]. sind) bei weitem nicht erreicht ${ }^{*}$. - Bemerkenswerter sind die $R Q$-Werte bei $2^{\circ}$ und $10^{\circ}$, nicht allein weil in diesem Bereich bisher kaum exakte Messungen durchgeführt worden zu sein scheinen, als vielmehr auch in Anbetracht ihrer Tiefe, die bei Getreidefrüchten bis jetzt wohl noch nicht beobachtet worden ist.

Vergleichbar sind allein gewisse Befunde von P lat enius ${ }^{29}$, der die Atmung von zehn Gemüsearten nach verschieden langer Lagerung bei $24^{\circ}$, $10^{\circ}$ und $0,5^{\circ} \mathrm{C}$ untersuchte und nur bei Kartoffeln bei $0,5^{\circ} R Q$-Werte feststellte, die denen unserer

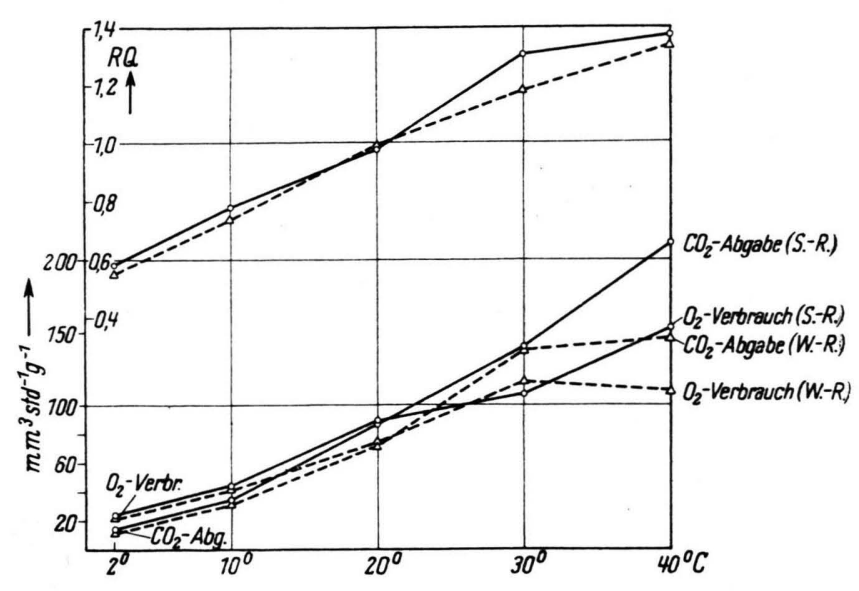

Abb. 1. $\mathrm{CO}_{2}$-Abgabe, $\mathrm{O}_{2}$-Verbrauch (beide in $\mathrm{mm}^{3}$ pro Stde. und $g$ Trockengewicht) und $R Q$ von Caryopsen des Sommer- $(-\bigcirc-)$ und des Winterroggens (-- $\triangle--)$ bei verschiedenen Temperaturen nach 23 -stdg. Quellung bei $23^{\circ} \mathrm{C}$.

Caryopsen bei $2^{\circ}$ gleichen (nämlich 0,45 nach 1-tägiger, 0,58 nach 11-15-tägiger, 0,56 nach $41-60$ tägiger und 0,66 nach $61-100$-tägiger Lagerung bei $\left.0,5^{\circ}\right)^{* *}$. Relativ niedrige $R Q$-Werte (um 0,8 ) fand $\mathrm{Pl}$ at enius auch noch beim Spinat bei $0,5^{\circ}$, während die der übrigen Objekte (Spargel, Erbsen, Bohnen, Möhren, Salat, Tomaten, Gurken) bei $0,5^{\circ}$ durchweg zwischen 0,9 und 1,07 lagen.

$\mathrm{Pl}$ at enius zieht aus seinen Befunden den naheliegenden Schluß, daß bei Kartoffeln und Spinat bei

** Die von P l a t e n i u s für die Atmungsintensität ermittelten Werte lassen sich mit den unsrigen nicht vergleichen, da jene auf das jeweilige Frischgewicht bezogen sind.

27 G. B onnier u. L. M a ngin, Ann. Sci. natur., Bot., Sér. 6, 18, 293-361 [1884].

28 G. T. H a r r ing $\mathrm{t}$ o $\mathrm{n}$, J. agric. Res. 23, 117-130 [1923].

29 H. Plat enius, Plant Physiol. 17, 179-197 [1942]. 


\begin{tabular}{|c|c|c|c|c|c|}
\hline & $2^{\circ} \mathrm{C}$ & $10^{\circ} \mathrm{C}$ & $20^{\circ} \mathrm{C}$ & $30^{\circ} \mathrm{C}$ & $40^{\circ} \mathrm{C}$ \\
\hline $\mathrm{S}$ & 10,223 & 9,66 & 1,8 & $-21,9$ & $-58,3$ \\
\hline W & 9,925 & 11,28 & 1,1 & $-20,5$ & $-36,4$ \\
\hline
\end{tabular}

Tab. 2. Differenz $\mathrm{O}_{2}$-Verbrauch minus $\mathrm{CO}_{2}$-Abgabe (in $\mathrm{mm}^{3}$ pro Stde. und $\mathrm{g}$ Trockengewicht) von Caryopsen des Sommer- (S) und des Winterroggens (W) bei $2-40^{\circ}$ nach 23-stdg. Quellung bei $23^{\circ} \mathrm{C}$.

$0,5^{\circ}\left(\mathrm{O}_{2}\right.$-reiche $)$ organische Säuren gebildet werden, was auch bei den Roggen-Caryopsen der Fall sein dürfte. Hierfür spricht z. B., daß diese bei 2 und $10^{\circ}$ nicht nur die relativ (d.h. im Verhältnis zum freiwerdenden $\mathrm{CO}_{2}$ ) höchste $\mathrm{O}_{2}$-Menge verbrauchen, sondern daß auch die Differenz $\mathrm{O}_{2}$-Verbrauch minus $\mathrm{CO}_{2}$-Abgabe hier ihre Maximalwerte erreicht (vgl. Tab. 2). Der Unterschied zwischen Sommer- und Winterroggen ist hier nur bei $10^{\circ}$ gesichert $(P=0,0008)$, während die $P$-Werte des Unterschiedes zwischen $2^{\circ}$ und $10^{\circ} 0,2$ (beim Sommer-) und 0,033 (beim Winterroggen) betragen. Die Lage des Maximums der $\mathrm{O}_{2}$ - $\mathrm{CO}_{2}$-Differenz der WinterroggenCaryopsen bei $10^{\circ}$ läßt vermuten, daß es sich hier um einen Kälteeffekt handelt, der mit der Vernalisation in keinem direkten Zusammenhang steht; stellen doch $10^{\circ}$ eine schlechtere Vernalisations-Temperatur dar als $2^{\circ}$.

$\mathrm{Da}$ durch tiefe Temperaturen die $\mathrm{CO}_{2}$-Produktion stärker gehemmt wird als der $\mathrm{O}_{2}$-Verbrauch, geht auch aus den $Q_{10}$-Werten hervor, die in Tab. 3 zusammengestellt sind und keinen grundlegenden Unterschied zwischen Sommer- und Winterroggen erkennen lassen.

\section{Isolierte Embryonen}

$\mathrm{Da}$ an der Atmung ganzer Caryopsen natürlich auch das Endosperm beteiligt, für den Vernalisations-Effekt aber allein der Embryo verantwortlich ist, wurden weitere Untersuchungen an isolierten Embryonen ausgeführt.

\section{a) Methode}

Hierzu wurden unter der binokularen Lupe mit Hilfe eines Skalpells, das vor jeder Präparation abgeflammt wurde, einige tausend Embryonen beider Roggenrassen

* Prochorova und Kretovi č hatten für die Atmungsintensität „des Korns“ im Bereich von $0-10^{\circ}$ $Q_{10}$-Werte zwischen 5 und 12 erhalten.

30 A. P. Prochorova u. V. L. Kretovič, Dokl. Akad. Nauk SSSR., N.S. 69, 401-403 [1949]; zit. n. Ber. wiss. Biol. 73, 32 [1951].

\begin{tabular}{|c|c|c|c|c|c|}
\hline & $2-10^{\circ} \mathrm{C}$ & $10-20^{\circ} \mathrm{C}$ & $20-30^{\circ} \mathrm{C}$ & $30-40^{\circ} \mathrm{C}$ \\
\hline $\mathrm{CO}_{2^{-}}$ & $\mathrm{S}$ & $2,97^{*}$ & 2,53 & 1,62 & 1,51 \\
Abgabe & $\mathrm{W}$ & 3,16 & 2,30 & 1,90 & 1,06 \\
\hline $\mathrm{O}_{2^{-}}$ & $\mathrm{S}$ & 2,07 & 2,03 & 1,22 & 1,42 \\
Verbrauch & $\mathrm{W}$ & 2,23 & 1,73 & 1,60 & 0,93 \\
\hline
\end{tabular}

Tab. 3. $Q_{10}$ der $\mathrm{CO}_{2}$-Abgabe und des $\mathrm{O}_{2}$-Verbrauchs von Caryopsen des Sommer- (S) und des Winterroggens (W) bei $2-40^{\circ}$ nach 23 -stdg. Quellung bei $23^{\circ} \mathrm{C}$.

isoliert, und zwar so, daß das Scutellum beim Endosperm verblieb ${ }^{*}$. Öfters waren Embryonen von Pilzmycelien durchwachsen und wurden verworfen, wenn die Infektion unter der Lupe zu erkennen war. Auf eine Sterilisation mußte leider verzichtet werden, da die Embryonen auf Desinfektionsmittel mit einer starken Herabsetzung der Atmungsintensität reagierten.

Je 20 Embryonen wurden in lufttrockenem Zustand gewogen und in Reaktionsgefäße mit je $2,0 \mathrm{~cm}^{3}$ dest. und sterilisierten Wassers gegeben, in denen sie bis zum eigentlichen Versuchsbeginn 1 Stde. lang bei der vorgesehenen Versuchstemperatur unter Schütteln gequollen wurden. Zur $\mathrm{O}_{2}$-Absorption und $\mathrm{CO}_{2}$-Freisetzung dienten je $0,3 \mathrm{~cm}^{3} n . \mathrm{KOH}$ bzw. $n$. HCl. Im übrigen wurde wie bei ganzen Caryopsen verfahren (s. o.), nur mit dem Unterschied, daß die Versuchsdauer bei $2^{\circ} 16$ Stdn., bei $10^{\circ}$ und $20^{\circ}$ dagegen $8 \mathrm{Stdn}$. betrug, daß - ebenso wie bei allen folgenden Versuchen - eine Schüttelfrequenz von 90 Doppelschlägen pro Min. gewählt und daß die Meßwerte auf das jeweilige Gewicht der lufttrockenen Embryonen bezogen wurden.

\section{b) Ergebnis se}

Die Atmungsintensitäten der Sommer- und Winterroggen-Embryonen bei gleicher Temperatur lassen sich nicht ohne weiteres vergleichen, da die Werte für $\mathrm{O}_{2}$-Verbrauch und $\mathrm{CO}_{2}$-Abgabe der isolierten Embryonen trotz Lagerung bei $2^{\circ * *}$ im Laufe der Zeit langsam absanken ${ }^{* * *}$. Es kann daher nur gesagt werden, daß die $\mathrm{CO}_{2}$-Abgabe (für Sommer- und Winterroggen zusammengenommen) durchschnittlich in runden Werten bei $2^{\circ} 180$, bei $10^{\circ} 870$ und bei $20^{\circ} 3300 \mathrm{~mm}^{3}$ Stdn. $^{-1} \mathrm{~g}$ (lufttrocken) ${ }^{-1}$ betrug, der $\mathrm{O}_{2}$-Verbrauch dagegen bei $2^{\circ} 215$, bei $10^{\circ} 1075$ und bei $20^{\circ} 3500 \mathrm{~mm}^{3}$ pro Stde. und g.

* Nach den Befunden von Pu rvis ${ }^{31}$ hat das Scutellum offenbar keinen maßgeblichen Einfluß auf den Vernalisations-Effekt angemessener Temperaturen.

** Bei lufttrockenen Embryonen (Wassergehalt etwa $15 \%$ ) haben $2^{\circ}$ keine vernalisierende Wirkung.

*** $\mathrm{Da}$ sich diese Versuchsserie zeitlich mit den beiden folgenden (vgl. Abschnitt IB 1 und 2) überschnitt, war nicht zu vermeiden, daß sich die Messungen über mehrere Monate erstreckten.

31 O. N. Pu rvis, Nature [London] 145, 462 [1940]. 
Die $\mathrm{CO}_{2}$-Produktion isolierter Embryonen ist demnach (wieder in runden Zahlen) bei $2^{\circ} 12,6$-mal so stark wie bei Sommer- und 14,7-mal so stark wie bei Winterroggen-Caryopsen, bei $10^{\circ} 25,6$-mal so stark wie bei Sommer- und 28,3-mal so stark wie bei Winterroggen-Caryopsen und endlich bei $20^{\circ} 38,4-$ mal so stark wie bei Sommer- und 46-mal so stark wie bei Winterroggen-Caryopsen. Entsprechend ist der $\mathrm{O}_{2}$-Verbrauch der Embryonen bei $2^{\circ}$ 8,8-mal so stark wie bei Sommer- und 9,7-mal so stark wie bei Winterroggen-Caryopsen, bei $10^{\circ} 24,6$-mal so stark wie bei Sommer- und 25,6-mal so stark wie bei Winterroggen-Caryopsen und schließlich bei $20^{\circ}$ 40-mal so stark wie bei Sommer- und 48-mal so stark wie bei Winterroggen-Caryopsen.

Dabei ist nicht einmal berücksichtigt, daß die Meßwerte bei Caryopsen auf das Gewicht nach 4-stdg. Trocknung bei $105^{\circ}$, bei den Embryonen dagegen auf das Gewicht in lufttrockenem Zustand bezogen ist. Hierbei ist ergänzend zu erwähnen, daß sich bei den Wägungen der Embryonen überraschenderweise eine deutliche Differenz zwischen Sommerund Winterroggen ergab: Das Gewicht von $20 \mathrm{Em}$ bryonen betrug bei ersterem durchschnittlich 14,9 , bei letzterem 13,05 mg. Es waren 1160 Sommerund 940 Winterroggen-Embryonen gewogen worden. Die Differenz ist mit $t_{103}=19,3$ und $P \ll 0,0002$ ausgezeichnet gesichert.

Da die Atmungsintensitäten der Embryonen wegen ihrer allmählichen Abnahme statistisch nicht getrennt bearbeitet wurden (vgl. den Anfang dieses Abschnittes), sind die Unterschiede der Proportionalitäts-Faktoren zwischen Sommer- und Winterroggen von untergeordneter Bedeutung. Interessanter ist, daß der Proportionalitäts-Faktor mit höherer Temperatur steil ansteigt, und zwar beim $\mathrm{O}_{2}$-Verbrauch noch mehr als bei der $\mathrm{CO}_{2}$-Produktion. Zum Vergleich sei darauf hingewiesen, daß Weizenembryonen nach $\mathrm{K}$ a rchewsky ${ }^{32}$ 12-mal, nach Burlakow ${ }^{33}$ 16-mal so stark atmen wie ganze Caryopsen und 20-mal so stark wie das Endosperm.

Bemerkenswert ist ferner, daß die $Q_{10}$-Werte der Atmungsintensität bei unseren Roggenembryonen wesentlich höher liegen als bei den Caryopsen: Der $Q_{10}$ beträgt im einzelnen für die $\mathrm{CO}_{2}$-Abgabe rund 7,2 bei $2-10^{\circ}$ und 3,8 bei $10-20^{\circ}$, für den $\mathrm{O}_{2}$-Verbrauch 7,5 bei $2-10^{\circ}$ und 3,1 bei $10-20^{\circ}$.

Im Gegensatz zur Atmungsintensität lie $\beta$ der $R Q$

32 K a rchewsky, Mem. Warsaw Univ. 9 [1901]; zit. n. Lehmann und Aichele, Keimungsphysiologie der Gräser (Gramineen). Stuttgart 1931.

\begin{tabular}{|c|cc|cc|cc|}
\hline & $2^{\circ} \mathrm{C}$ & \multicolumn{2}{|c|}{$10^{\circ} \mathrm{C}$} & \multicolumn{2}{|c|}{$20^{\circ} \mathrm{C}$} \\
\hline $\mathrm{S}$ & 0,834 & $(5)$ & 0,931 & $(1)$ & 0,972 & $(2)$ \\
$\mathrm{W}$ & 0,790 & $(3)$ & 0,825 & $(1)$ & 0,906 & $(2)$ \\
\hline$P$ & 0,020 & - & 0,1 \\
\hline
\end{tabular}

Tab. 4. $R Q$ von Embryonen des Sommer- (S) und des Winterroggens (W) bei $2^{\circ}, 10^{\circ}$ und $20^{\circ} \mathrm{C}$ nach l-stdg. Vorquellung bei Versuchstemperatur. In Klammern jeweils die Zahl der Parallelversuche.

der isolierten Embryonen keinen Einfluß der Lagerungsdauer erkennen, so daß hier eine Trennung nach Sommer- und Winterroggen ohne weiteres durchführbar war. Die dabei erzielten Ergebnisse entsprechen weitgehend den bei ganzen Caryopsen erhaltenen, wie aus Tab. 4 hervorgeht:

Stets lag der $R Q$ bei den Embryonen des Winterroggens im Durchschnitt tiefer als bei denen des Sommerroggens, und zwar im einzelnen bei $2^{\circ}$ und $10^{\circ}$ höher und bei $20^{\circ}$ tiefer als in den entsprechenden Versuchen mit ganzen Caryopsen. Das totale $P$ beträgt hier 0,007 , wobei nur die Werte für $2^{\circ}$ und $20^{\circ}$ berücksichtigt sind.

\section{B. Langfristige Versuche bei $+2{ }^{\circ} \mathrm{C}$}

Die im vorhergehenden geschilderten kurzfristigen (6-16-stdg.) Atmungsuntersuchungen an Caryopsen und Embryonen hatten durchweg das Ergebnis, daß bei den angewandten Temperaturen $\left(2^{\circ}\right.$ bis $40^{\circ} \mathrm{C}$ ) der $R Q$ des Winterroggens tiefer liegt als der des Sommerroggens. Nun entsprach die Dauer dieser Versuche aber bei weitem nicht der Dauer einer wirksamen Vernalisations-Behandlung. Es lag daher nahe, weitere Versuche von der Dauer einer wirksamen Vernalisations-Behandlung durchzuführen.

\section{Ganze Caryopsen}

a) Methode

Petri-Schalen wurden mit $5 \mathrm{~g}$ lufttrockener Caryopsen (etwa 150 Stück) des Sommer- oder des Winterroggens beschickt. Diese wurden 1 Stde. lang mit $8 \mathrm{~cm}^{3}$ einer 3-proz. Perhydrol-Lösung (unter Zusatz einiger Tropfen einer Saponin-Lösung) oberflächlich desinfiziert, anschlieBend 2-mal mit sterilem Wasser abgespült, mit einem Stück Filtrierpapier je Schale unterlegt, mit $6 \mathrm{~cm}^{3}$ sterilen Wassers versetzt und in einen Kühlschrank (Temperatur $+2 \pm 1 / 2^{\circ} \mathrm{C}$ ) gebracht.

In Abständen von etwa einer Woche wurde dem Kühlschrank an einem Tage eine Petri-Schale mit Sommer-,

33 G. B u rlakow, Arb. Naturf. Ges. Charkow 31, Beil., 1-15 [1897]; zit. n. L e h m a n und A i chele. 
am nächsten oder übernächsten Tag eine solche mit Winterroggen-Material entnommen, mit dem im Kältelabcratorium bei etwa $0^{\circ}$ die wiederum je $2,4 \mathrm{~cm}^{3}$ sterilen Wassers enthaltenden Reaktionsgefäße des WarburgApparates gefüllt wurden. In Anbetracht der im Laufe der Kältebehandlung beträchtlich steigenden Atmungsintensität (s. u.), der einsetzenden Keimung und des fortschreitenden Wachstums der Keimlinge wurden von einer Woche zur anderen immer weniger, möglichst einheitliche Caryopsen bzw. Pflänzchen mit anhängender Frucht je Gefäß verwandt, zuletzt (nach 5 Wochen) nur noch vier, deren Coleoptilen knapp 3 und deren Wurzeln bis zu $7 \mathrm{~cm}$ lang waren. Selbstverständlich wurde größter Wert darauf gelegt, daß die Keimlinge möglichst unversehrt in die Reaktionsgefäße gelangten. - Trotz der Desinfektion bei Versuchsbeginn waren einzelne Caryopsen von Schimmelpilzen befallen, die vermutlich aus den Embryonen (s. o.) hervorgewachsen waren und nach Entnahme der Caryopsen aus der Petri-Schale sofort kollabierten; die verpilzten Früchte wurden verworfen. Die Atmungsmessungen wurden bei der VernalisationsTemperatur $\left(+2{ }^{\circ} \mathrm{C}\right)$ vorgenommen und dauerten wie bei den kurzfristigen Versuchen je 16 Stunden.

22 Tage nach Inangriffnahme dieses Reihenversuches wurde ein zweiter begonnen, der sich von dem ersten nur dadurch unterschied, daß von je zwei zusammengehörigen Atmungsmessungen die erste am Winter- und die zweite am Sommerroggen durchgeführt wurde. Nach Beendigung eines jeden Einzelversuches wurden die Caryopsen (ggf. nebst Keimlingen) in gewohnter Weise (vgl. Abschnitt I A 1 a) getrocknet und gewogen. - Im folgenden Abschnitt werden stets die Durchschnittswerte beider Meßreihen wiedergegeben, während die graphische Darstellung der Atmungsintensitäten auch die Einzelwerte ausweist.

\section{b) Ergebnisse}

Bei unseren Atmungsmessungen an Caryopsen während einer 5-wöchigen Vernalisations-Behandlung fällt zunächst die starke Zunahme der Intensität ins Auge, die für den $\mathrm{O}_{2}$-Verbrauch beim Sommerroggen über $1600 \%$, beim Winterroggen über $900 \%$ betrug. Diese Intensitätssteigerung war vor allem durch den relativ hohen Quellungsgrad bedingt, der auch die Keimung ermöglichte. Dies geht z. B. aus einem Vergleich mit den Atmungsmessungen von $\mathrm{Met}^{34}$ an Winterweizen während 4-wöchiger Vernalisation hervor. Met hatte je $200 \mathrm{~g}$ WinterweizenCaryopsen vor Vernalisations-Beginn mit nur $80 \mathrm{~cm}^{3}$ Wasser 2 Tage lang bei $16,5^{\circ} \mathrm{C}$ quellen lassen, wodurch die Keimung verhindert und nur eine Steigerung der $\mathrm{CO}_{2}$-Abgabe von etwa $14 \mathrm{~mm}^{3}$ pro Stde. und $g$ Trockengewicht am 1. Vernalisations-Tag auf 33 am 29. ermöglicht wurde.

34 M. Met, Étude de la respiration du Blé au cours d'une germination normale et pendant le traitement de vernalisation. 26 pp. Paris 1950.

\begin{tabular}{|c|c|c|c|c|c|c|}
\hline & $\begin{array}{c}1-2 \\
\text { Tage }\end{array}$ & $\begin{array}{c}1 \\
\text { Woche }\end{array}$ & $\begin{array}{c}2 \\
\text { Wochen }\end{array}$ & $\begin{array}{c}3 \\
\text { Wochen }\end{array}$ & $\begin{array}{c}4 \\
\text { Wochen }\end{array}$ & $\begin{array}{c}5 \\
\text { Wochen }\end{array}$ \\
\hline $\mathrm{S}$ & 17 & 42 & 78 & 132 & 226 & 298 \\
$\mathrm{~W}$ & 14 & 33 & 69 & 99 & 142 & 144 \\
\hline$P$ & 0,050 & 0,054 & 0,046 & 0,0145 & 0,00049 & 0,0011 \\
\hline
\end{tabular}

Tab. 5. Statistische Sicherung des Unterschiedes im $\mathrm{O}_{2}$ Verbrauch (in $\mathrm{mm}^{3}$ pro Stde. und g Trockengewicht) zwischen Caryopsen des Sommer- und des Winterroggens bei $2 \circ \mathrm{C}$ während einer 5-wöchigen Kältebehandlung. Zwei Parallel-Meßreihen.

Leider verzichtete $\mathrm{M}$ e $\mathrm{t}$ darauf, das Verhalten von Sommer- und Winterweizen unter VernalisationsBedingungen $\mathrm{zu}$ vergleichen *; vielmehr untersuchte er das des Sommerweizens nur bei 16,5 und 25 bis $28^{\circ} \mathrm{C}$. Wie unsere Versuche mit Roggen nun zeigen, prägt sich der auch schon anfangs (vgl. Abschn. I A 1 b) beobachtete Intensitätsunterschied zwischen Sommerund Winterform im Verlauf der Kältebehandlung immer stärker aus (vgl. Abb. 2); die statistische Sicherung dieser Differenz im $\mathrm{O}_{2}$-Verbrauch geht aus Tab. 5 hervor. Das totale $P$ beläuft sich hier auf $6,25 \cdot 10^{-9}$. Ähnliches trifft für die $\mathrm{CO}_{2}$-Abgabe zu. $\mathrm{Da} \beta$ die Werte bei Versuchsbeginn $\left(\mathrm{O}_{2}\right.$-Verbrauch 17 bzw. $14 \mathrm{~mm}^{3}$ Stdn $^{-1} \mathrm{~g}^{-1}$ ) tiefer liegen als in den entsprechenden kurzfristigen Versuchen (Tab. 1), ist auf die unterschiedliche Vorbehandlung (hier Perhydrol-Desinfektion und Quellung bei $2^{\circ}$ mit geringerer Wassermenge) zurückzuführen.

Was den $R Q$ anlangt, so ist allgemein ein gewisser Anstieg während der Vernalisations-Zeit zu beobachten, und zwar von 0,636 beim Sommer- und 0,609 beim Winterroggen auf 0,746 bzw. 0,726 nach $35 \mathrm{Ta}$ gen $(P=0,0015$ für Sommer- und 0,024 für Winterroggen; totales $P=0,0001)$. Dieser Befund harmoniert wiederum mit den Feststellungen von $\mathrm{Met}$, der beim Winterweizen unter den oben geschilderten Bedíngungen gleichfalls einen $R Q$-Anstieg (und zwar von 0,78 auf 0,94$)$ gefunden hatte.

Weniger gut ist bei unseren hier in Rede stehenden Versuchen die $R Q$-Differenz zwischen beiden Roggenrassen zu sichern. Nur bei den Messungen $1-2$ Tage und 4 und 5 Wochen nach Vernalisations-

* Dasselbe gilt für Sisakjan und Filippovič 35 , die ihre Atmungsmessungen auf Winterweizen und Wintergerste beschränkten und ein Intensitätsmaximum (das sie als Übergang zur Lichtphase betrachten) am 46. bzw. 33. Vernalisations-Tag fanden.

35 N. M. S is a kj a n u. I. I. F i li p povič, Dokl. Akad. Nauk SSSR., N.S. 76, 443-446 [1951]; zit. n. Ber. wiss. Biol. 73, 294 [1951]. 
Beginn war im Durchschnitt ein deutlicherer Unterschied (in der gewohnten Richtung) zu erkennen, der ein totales $P$ von 0,0205 ergab.

\section{Isolierte Embryonen}

\section{a) Methode}

Unsere Methode war auch bei diesen Versuchen im wesentlichen die im Abschnitt IA 2 a geschilderte, nur mit dem Unterschied, daß in je einem Teil der zur Messung der Differenz $\mathrm{O}_{2}$-Verbrauch minus $\mathrm{CO}_{2}$-Abgabe benutzten Reaktionsgefäße 16 bzw. 64 Stdn. nach Versuchsbeginn oder erst bei Versuchsende $0,3 \mathrm{~cm}^{3} n . \mathrm{HCl}$ zur Freisetzung der bis dahin retinierten $\mathrm{CO}_{2}$-Menge eingekippt wurden. Das bei Versuchsschlu $ß$ freigesetzte $\mathrm{CO}_{2}$-Quantum wurde bei der Auswertung der Messungen gleichmäßig auf die seit der 17. bzw. 65. Versuchsstunde abgelaufene Zeit aufgeschlüsselt. Mit Sommerund Winterroggen-Embryonen wurden je zwei gleichartige Versuchsreihen durchgeführt.

\section{b) Ergebnisse}

Wie aus Abb. 3 hervorgeht, nimmt die Atmungsintensität anfangs ständig zu, und zwar - ebenso wie bei den ganzen Caryopsen - beim Sommerroggen in noch stärkerem Grade als beim Winterroggen. Dieser Anstieg hält bei den SommerroggenEmbryonen aber nur etwa bis zum 6. Tage an; von da ab bleiben $\mathrm{CO}_{2}$-Produktion und $\mathrm{O}_{2}$-Verbrauch etwa konstant und erreichen etwa am 13. Tag mit rund 400 bzw. $450 \mathrm{~mm}^{3}$ je g und Stde. ein flaches Maximum, dem ein allmählicher Abfall folgt.

Beim Winterroggen dagegen steigt die Intensität auch nach dem 6 . Tage zunächst kontinuierlich weiter an, übertrifft etwa vom 7 . Tage ab die des Sommerroggens und erreicht gleichfalls am 13. Tag ihren Höhepunkt bei rund 550 bzw. $607 \mathrm{~mm}^{3}$ je g und Stunde. Anschließend sinken die Werte wieder $a b$, und zwar stärker als die des Sommerroggens, die etwa vom 16. Tag ab wieder relativ höher liegen. Gegen Versuchsschluß (nach etwa 30 Tagen) gleichen sich die Atmungsintensitäten beider Roggensorten einander an $\left(\mathrm{CO}_{2}\right.$-Produktion etwa $105, \mathrm{O}_{2}$-Verbrauch rund $140 \mathrm{~mm}^{3}$ je $\mathrm{g}$ und Stunde).

Für eine durchgehende statistische Sicherung des Unterschiedes zwischen Sommer- und Winterroggen reichen je zwei Meßreihen (deren Mittelwerte in Abb. 3 dargestellt sind) nicht aus, da die Ergebnisse zusammengehöriger Reihen stets ein wenig differierten, was auch hier mit der Dauer der vorangegangenen Lagerung zusammenhängen dürfte. Mit zunehmender Lagerzeit sank und verzögerte sich nämlich bei beiden Roggensorten das Intensitätmaximum ein wenig, und die darauffolgende Abnahme der Intensität verlangsamte sich entsprechend.

Die Bewegung des $R Q$ beginnt für Sommer- und für Winterroggen mit einem deutlichen Abfall von 0,862 auf 0,810 bzw. von 0,804 auf 0,765 in den

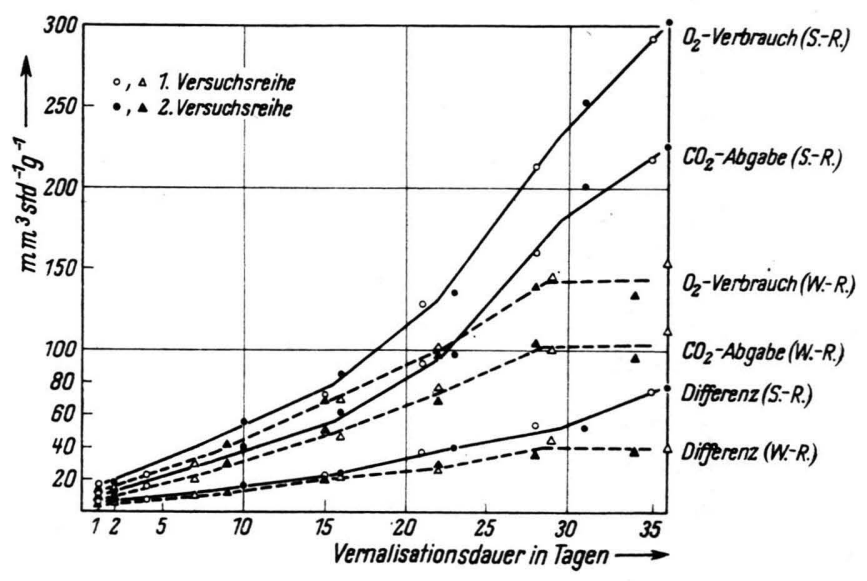

Abb. 2. Atmungsintensität bei Caryopsen des Sommer$(\bigcirc, \bullet)$ und des Winterroggens $(\triangle, \Delta)$ im Verlauf einer 5-wöchigen Vernalisations-Behandlung $\left(+2^{\circ} \mathrm{C}\right)$.

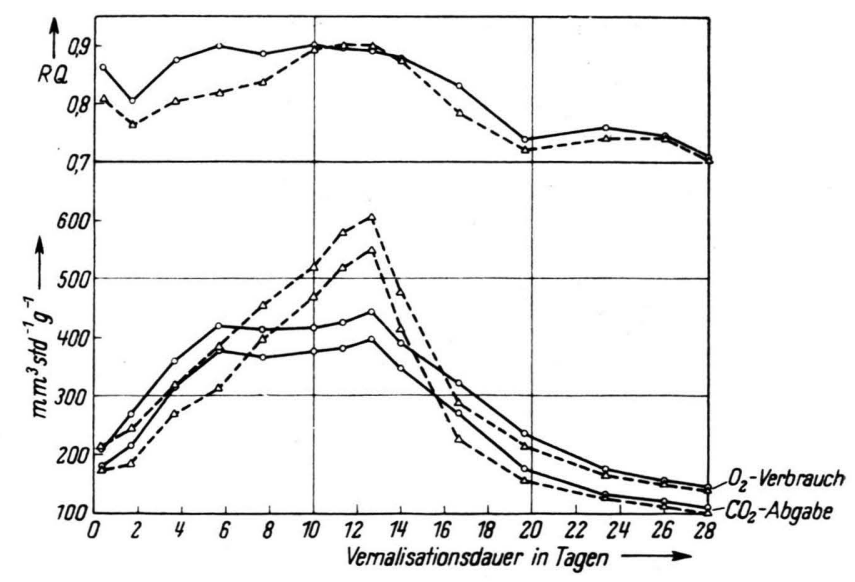

Abb. 3. Atmungsverlauf bei isolierten Embryonen des Sommer- $(-\bigcirc-)$ und des Winterroggens (-- $\triangle--)$ während einer 4 -wöchigen Kältebehandlung $\left(+2^{\circ} \mathrm{C}\right)$. Oben $R Q$, unten $\mathrm{CO}_{2}$-Abgabe und $\mathrm{O}_{2}$-Verbrauch. Die Kurven sind aus den Mittelwerten von je zwei Parallelreihen gebildet.

ersten 2-3 Tagen, der statistisch gut gesichert ist $(P=0,05$ für Sommer-, $P=0,0082$ für Winterroggen, totales $P=0,0018$ ). Bei beiden folgt dann ein beim Sommerroggen schnellerer, beim Winterroggen langsamerer Anstieg auf $0,9(P=0,0023$ bzw. 0,001$)$. Parallel zur Atmungsintensität sinkt auch der $R Q$ wieder vom 13. Tage ab und schwankt vom 19. Tag 
bis zum Versuchsschluß zwischen 0,7 und 0,75. (Auch der neuerliche Abfall ist mit $P=0,0019$ für Sommer- und 0,002 für Winterroggen gut gesichert.) Dieser letzte Befund läßt darauf schließen, daß nach Verbrauch der Kohlenhydrate, d. h. nach Überschreitung des Intensitätsmaximums, vor allem Fette veratmet werden.

Auf die Übereinstimmung zwischen der in diesem Versuch bis zum Intensitäts- und RQ-Maximum unserer Embryonen verstrichenen Zeit einerseits und der gleichfalls etwa 14-tägigen „lag period“ ( $\mathrm{P} \mathrm{u} \mathrm{r}$ v i s ${ }^{10}$ ) andererseits werden wir auf S. 228 eingehen.

\section{Kurzfristige Untersuchungen an Arabidopsis thaliana (L.) HeYNH.}

Zur Überprüfung der im Abschnitt I A erhobenen Befunde auf ihre Gültigkeit bei anderen Pflanzen wurden kurzfristige Atmungsmessungen auch an den Samen je einer sommer- und winterannuellen Rasse der Gänserauke (Arabidopsis thaliana) durchgeführt. L a ib a ch ${ }^{36}$ hatte nämlich neben zahlreichen sommereinjährigen Arabidopsis-Rassen auch zwei winterannuelle (,Stockholm“ und „Kopenhagen") ausfindig gemacht, die zu denjenigen Cruciferen gehören, welche sich schon auf dem Samenstadium vernalisieren lassen. Gegenüber den zu Vernalisations-Studien z. Z. meist benutzten Roggenund Weizensorten zeichnet sich die Langtagpflanze Arabidopsis thaliana unter anderem durch Kürze der Vegetationsdauer, Anspruchslosigkeit in bezug auf Platz und Boden sowie reichliche Produktion von Samen aus, die im wesentlichen aus dem Embryo bestehen. So ist damit zu rechnen, daß Arabidopsis künftig eine gewisse Rolle in der Physiologie der Blütenbildung spielen wird; es erschien daher sinnvoll, unsere Untersuchungen gerade auf dieses $\mathrm{Ob}$ jekt auszudehnen *.

\section{a) Methode}

Samen der Rassen Limburg ${ }_{5}\left(L i_{5}\right.$, sommerannuell) und Stockholm (St, winterannuell) wurden in Mengen von ungefähr $25 \mathrm{mg}$ (für Messungen bei $20^{\circ}$; bei höherer Temperatur weniger, bei tieferer mehr) abgewogen und in Warburg-Reaktionsgefäße gegeben. Dort wurden ihnen 30 Min. vor Versuchsbeginn je $2 \mathrm{~cm}^{3}$ sterilen Wassers zugesetzt, mit dem sie innerhalb der letzten Viertelstunde vor Versuchsbeginn im Wasserbad des WarburgApparates unter Schütteln auf die Versuchstemperatur gebracht wurden. Wie bei isolierten Roggen-Embryonen wurden je $0,3 \mathrm{~cm}^{3} \mathrm{KOH}$ bzw. $\mathrm{HCl}$ benutzt. Die Ver-

36 F. L a i b a c h, Naturwissenschaften 31, 246 [1943]; Beitr. Biol. Pfl. 28, 173-210 [1951]. suchsdauer betrug bei $2^{\circ} \mathrm{C} 48$, bei $10^{\circ}, 20^{\circ}$ und $30^{\circ}$ dagegen 24 Stunden. Die Meßwerte wurden auf das Frischgewicht bezogen; der Wassergehalt lufttrockener Samen betrug etwa 6 Prozent.

\section{b) Ergebnis se}

Was die Atmungsintensität anlangt, so stimmen unsere Befunde an Arabidopsis im wesentlichen mit den am Roggen erhobenen überein:

Auch hier liegen $\mathrm{CO}_{2}$-Produktion und $\mathrm{O}_{2}$-Verbrauch bei allen angewandten Temperaturen $\left(+2^{\circ}, 10^{\circ}\right.$,

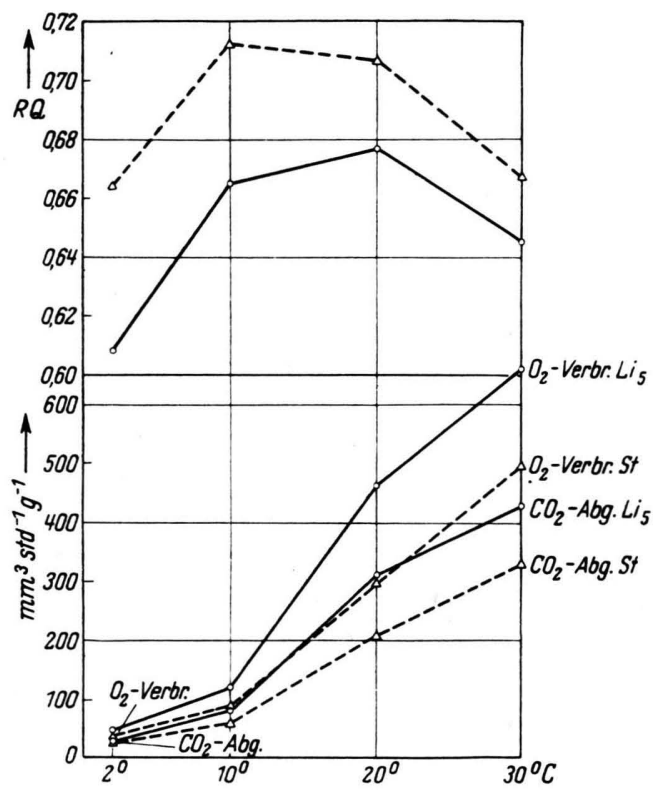

Abb. 4. $R Q, \mathrm{CO}_{2}$-Abgabe und $\mathrm{O}_{2}$-Verbrauch (in $\mathrm{mm}^{3}$ pro Stde. und g) von Samen der Arabidopsis-Rassen $\mathbf{L i}_{5}$ $(--)$ und St $(-\triangle-)$ bei $2-30^{\circ} \mathrm{C}$.

$20^{\circ}$ und $30^{\circ} \mathrm{C}$ ) bei der sommerannuellen Rasse $L i_{5}$ höher als bei der winterannuellen St (vgl. Abb.4). Wie Tab. 6 ausweist, ist die Differenz im allgemeinen gut gesichert; das totale $P$ beträgt für die $\mathrm{CO}_{2}$ Abgabe 0,0004 und $5 \cdot 10^{-7}$ für den $\mathrm{O}_{2}$-Verbrauch.

Die $Q_{10}$-Werte für die Steigerung der Atmungsintensität durch höhere Temperaturen sind in Tab. 7 zusammengefaßt. Diese zeigt, daß bei Arabidopsis - im Gegensatz zu den Verhältnissen beim Roggen - der $Q_{10}$ sein Maximum im allgemeinen zwischen $10^{\circ}$ und $20^{\circ}$ hat und bei der sommerannuellen Rasse zwischen $2^{\circ}$ und $20^{\circ}$ höher, zwischen $20^{\circ}$ und $30^{\circ}$ tiefer liegt als bei der wintereinjährigen. Im ganzen gesehen ist die Atmungsintensität der Arabidopsis-

* Für die freundliche Überlassung von Samenmaterial habe ich Herrn Prof. Dr. F. Laibach zu danken. 


\begin{tabular}{|c|c|c|c|c|c|}
\hline & & $2^{\circ} \mathrm{C}$ & $10^{\circ} \mathrm{C}$ & $20^{\circ} \mathrm{C}$ & $30^{\circ} \mathrm{C}$ \\
\hline \multirow{2}{*}{$\begin{array}{c}\mathrm{CO}_{2-}^{-} \\
\text {Abgabe }\end{array}$} & $\begin{array}{l}L i_{5} \\
S t\end{array}$ & $\begin{array}{l}25,8(3) \\
25,65(4)\end{array}$ & $\begin{array}{l}79,4(1) \\
61,3(1)\end{array}$ & $\begin{array}{l}313(3) \\
211(2)\end{array}$ & $\begin{array}{l}427(4) \\
330(2)\end{array}$ \\
\hline & $P$ & $>0,3$ & - & 0,0015 & 0,024 \\
\hline \multirow{2}{*}{$\begin{array}{c}\mathrm{O}_{2^{-}} \\
\text {Verbrauch }\end{array}$} & $\begin{array}{c}L i_{5} \\
S t\end{array}$ & $\begin{array}{l}42,4(3) \\
38,1(4)\end{array}$ & $\begin{array}{r}119,5(1) \\
86,3(1)\end{array}$ & $\begin{array}{ll}462 & (3) \\
298,5 & (2)\end{array}$ & $\begin{array}{l}660(4) \\
497(2)\end{array}$ \\
\hline & $P$ & 0,0023 & - & 0,0004 & 0,014 \\
\hline \multirow{2}{*}{$R Q$} & $\begin{array}{l}L i_{5} \\
S t\end{array}$ & $\begin{array}{l}0,608(3) \\
0,664(4)\end{array}$ & $\begin{array}{l}0,665(1) \\
0,713(1)\end{array}$ & $\begin{array}{l}0,677(3) \\
0,707(2)\end{array} \mid$ & $\begin{array}{l}0,645(4) \\
0,667(2)\end{array}$ \\
\hline & $P$ & 0,026 & - & 0,022 & 0,09 \\
\hline
\end{tabular}

Tab. 6. Statistische Sicherung der Unterschiede in $\mathbf{C O}_{2}$ Abgabe, $\mathrm{O}_{2}$-Verbrauch und $R Q$ von Samen der Rassen Limburg $_{5}$ (sommerannuell) und Stockholm (winterannuell) von Arabidopsis thaliana bei $2^{\circ}, 10^{\circ}, 20^{\circ}$ und $30^{\circ} \mathrm{C}$ nach $1 / 2$-stdg. Vorquellung bei Versuchstemperatur. In Klammern die Zahl der jeweiligen Parallelmessungen. P-Werte nach $\mathrm{P}$ ä $\mathrm{t}$ a u ${ }^{23}$.

Samen unter unseren Versuchsbedingungen etwa doppelt bis 3-mal so groß wie bei Roggen-Caryopsen, aber nur etwa $1 / 10$-mal so groß wie bei isolierten Roggen-Embryonen.

Wie bei der Veratmung von Fetten üblich, liegt der $R Q$ der Arabidopsis-Samen in der Größenordnung von 0,7 (vgl. Abb. 4). Auch hier zeigt sich ein ausgeprägter Unterschied zwischen sommer- und winterannueller Rasse, der aber in umgekehrter Richtung liegt als beim Roggen: Bei allen angewandten Temperaturen liegt hier der durchschnittliche $R Q$ der winterannuellen Rasse $S t$ höher als der von $L i_{5}$. (Die statistische Sicherung der Differenz geht aus Tab. 6 hervor; das totale $P$ beträgt 0,0008 .) Schon hier muß auf die Tatsache hingewiesen werden, daß der $R Q$ sein Maximum offenbar im Bereich zwischen $10^{\circ}$ und $20^{\circ}$ hat und nicht nur bei $2^{\circ}$, sondern auch bei $30^{\circ}$ deutlich tiefer liegt ${ }^{*}$. (Die RQ-Differenz zwischen $20^{\circ}$ und $30^{\circ}$ ist für $\mathrm{Li}_{5}$ mit $P=0,008$, für $\mathrm{St}$ mit $P=0,0012$ gesichert; $P_{\text {tot }}=0,00006$.) Während nun $2^{\circ}$ unbedingt vernalisierend wirken, tritt bei $30^{\circ}$ durchaus keine Vernalisation, sondern im Gegenteil Devernalisation ein $\left(\mathrm{Napp}-\mathrm{Z}\right.$ in $\left.\mathrm{n}^{8}\right)$.

Ein langfristiger (29-tägiger) Versuch nach Art der mit isolierten Roggen-Embryonen vorgenommenen wurde auch bei A.-Samen der Rasse Stockholm durchgeführt. Die Methode war dabei die gleiche, wie im Abschnitt II a geschildert. Die Atmungsintensität stieg während der

* Ähnliches ist m. W. bisher nur von Norris 37 an Zwiebelwurzeln beobachtet worden.

\begin{tabular}{|c|c|c|c|c|}
\hline & & $2-10^{\circ} \mathrm{C}$ & $10-20^{\circ} \mathrm{C}$ & $20-30^{\circ} \mathrm{C}$ \\
\hline \multirow{2}{*}{$\mathrm{CO}_{2}$-Abgabe } & $L i_{5}$ & 4,08 & 3,94 & 1,37 \\
& $\mathrm{St}$ & 2,97 & 3,44 & $\mathbf{1 , 5 5}$ \\
\hline \multirow{2}{*}{$\mathrm{O}_{2}$-Verbrauch } & $L i_{5}$ & 3,65 & 3,97 & 1,43 \\
& $\mathrm{~S} t$ & 2,78 & 3,46 & 1,67 \\
\hline
\end{tabular}

Tab. 7. $Q_{10}$ der $\mathrm{CO}_{2}$-Abgabe und des $\mathrm{O}_{2}$-Verbrauches von Samen der Arabidopsis-Rassen Limburg (sommerannuell) und $\mathrm{St}$ (winterannuell) bei $2-30^{\circ} \mathrm{C}$.

ersten 3 Wochen ständig an $\left(\mathrm{O}_{2}\right.$-Verbrauch von 37,5 auf $369 \mathrm{~mm}^{3}$ pro $\mathrm{g}$ und Stde.) und sank dann bis Versuchsschluß nur ganz geringfügig. Der $R Q$ stieg während der ersten 5 Tage von 0,643 auf 0,720 , sank dann bis zum 12. Tag auf 0,680 , stieg bis zum 19. wieder auf 0,80 und blieb von da ab bis zum Versuchsschluß etwa konstant.

\section{Diskussion der Ergebnisse}

Im allgemeinen wächst die Geschwindigkeit chemischer und physiologischer Vorgänge innerhalb gewisser Grenzen mit steigender Temperatur. Eine Ausnahme von dieser Regel macht bei bestimmten zweijährigen und winterannuellen Pflanzen die Blütenbildung, die in diesen Fällen gerade durch tiefe Temperaturen (etwa $+1-6^{\circ} \mathrm{C}$ ) schneller herbeigeführt wird als durch höhere.

Als erste versuchten $\mathrm{Lang}$ und $\mathrm{Melchers^{j }}$ (1947) - 1952 auch Purvis und Gregory ${ }^{7}$ diesès Paradoxon zu erklären, indem sie mindestens drei Teilvorgänge mit normaler Temperaturabhängigkeit $\left(Q_{10}>1\right)$ annahmen, die für die Erreichung (oder Nichterreichung) der Blühreife verantwortlich sein sollen: Im Verlauf des ersten wird - dieser Deutung zufolge - ein wärmeempfindliches Zwischenstadium erreicht, das erst in einem zweiten Prozeß in ein wärmebeständiges Endstadium überführt wird. Schon bei mittleren Temperaturen (etwa $20^{\circ} \mathrm{C}$ ) wird bei den (eben deshalb kältebedürftigen) Pflanzen der Zwischenzustand großenteils oder völlig durch einen dritten Vorgang zerstört, so daß es kaum oder gar nicht zur Anhäufung des für die Blütenbildung erforderlichen Endzustandes kommt. Bei Sommerannuellen, genauer bei den nicht kältebedürftigen Pflanzen, soll der labile Zwischenzustand in der Weise stabilisiert sein oder umgangen werden, daß der letzte Vorgang (III) nicht abläuft.

Nun sind die Vernalisations-Vorgänge (I und II) sauerstoffbedürftig (Gregory und $\mathrm{Purvis}^{9}$ ) und werden durch Glucosezugabe gefördert $\left(\mathrm{Purvis}{ }^{10}\right)$;

37 W. E. Norris jr., Biochim. biophysica Acta [Amsterdam] 7, 225-237 [1951]. 
die Devernalisation (Vorgang III) dagegen erfolgt auch unter anaeroben Bedingungen. Es lag daher die Annahme nahe, daß die Vorgänge I und II oxydativer, Vorgang III aber glykolytischer Natur seien. Anhaltspunkte für die Richtigkeit einer solchen Hypothese hätte möglicherweise der Vergleich des Gaswechsels kältebedürftiger mit dem nicht kältebedürftiger Pflanzen erbringen können.

So hätte es eine starke Stütze für die soeben angegebene Hypothese bedeutet, wenn in unseren Untersuchungen der $R Q$ der winterannuellen Rassen durch die Kältebehandlung stets dem $R Q$ der entsprechenden sommereinjährigen Formen (durch Senkung) angenähert worden wäre und normalerweise über diesem gelegen hätte, wie es bei Arabidopsis der Fall war. Wie die mitgeteilten Versuchsergebnisse aber zeigen, trifft dies nicht allgemein zu; denn bei Arabidopsis liegt der $R Q$ nicht nur im Bereich niedriger (vernalisierender) Wärmegrade, sondern auch bei höheren (devernalisierenden) Temperaturen tiefer als bei der ,indifferenten “ Temperatur von $20^{\circ}$.

Die Versuche mit Roggen bieten nur insoweit eine Deutungsmöglichkeit in dem oben genannten Sinne, als hier unter devernalisierenden Bedingungen der $R Q$ über 1 liegt, - nicht aber insofern, als hier der $R Q$ der Sommerform allgemein über dem der Winterform liegt, während es ja der Hypothese zufolge gerade umgekehrt sein müßte.

Dennoch erscheint es nützlich, die erhobenen Befunde mitzuteilen; denn erstens ergab die Durchsicht der Literatur, daß Gaswechsel-Untersuchungen an keimenden Samen mit modernen Mitteln in einem so weiten Temperaturbereich offenbar bisher noch nicht durchgeführt worden sind, und da die Ergebnisse - vor allem auch bezüglich des $R Q$ - den der Atmung zugrunde liegenden hauptsächlichen Speicherstoffen der Samen vollauf entsprechen, dürften auch die Änderungen des $R Q$ in Abhängigkeit von der Temperatur bei kohlenhydrat- und fettspeichernden Samen weiteren keimungsphysiologischen Arbeiten brauchbare Anknüpfungspunkte liefern.

Vor allem aber wurde ein Befund erhoben, der uns auch für die Physiologie der Vernalisation von unmittelbarer Bedeutung zu sein scheint. Während die Wirkungskurve der Vernalisation bei ganzen RoggenCaryopsen schon von den ersten Behandlungstagen an langsam ansteigt, haben bei isolierten Embryonen die beiden ersten Wochen einer Vernalisationsbe-

38 O. N. Purvis, Ann. Botany, N.S. 12, 183-206 [1948]. handlung - unabhängig von eventueller Zuckerzugabe - keinen am Blühalter feststellbaren Effekt (Purvis ${ }^{10,38}$. Gerade in dieser Phase der Unwirksamkeit der Kältebehandlung („lag period“) steigt aber die Atmungsintensität isolierter Roggen-Embryo-
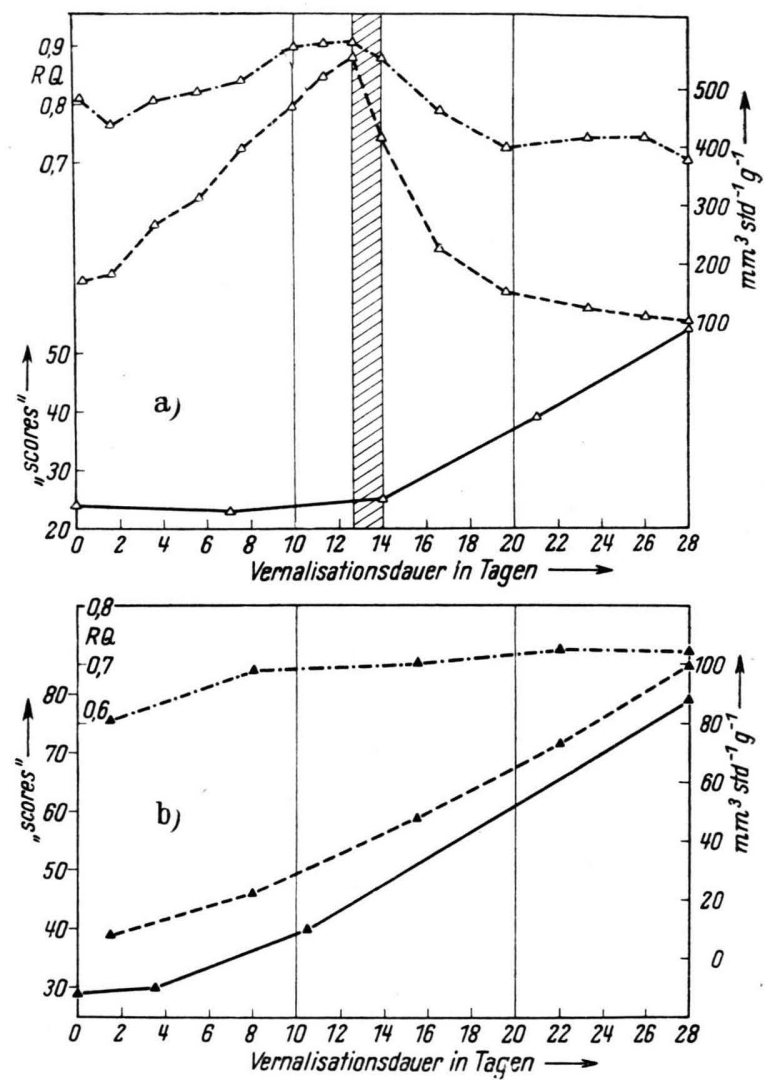

Abb. 5a. Isolierte Embryonen des Petkuser Winterroggens. Untere Kurve $(-\triangle-)$ Vernalisations-Effekt in willkürlichen Einheiten (,scores“) in Abhängigkeit von der Dauer einer Kältebehandlung bei $+1^{\circ} \mathrm{C}$ (nach Purvis ${ }^{38}$, Seite 187). Mittlere Kurve $\left(-\triangle-_{--}\right) \mathrm{CO}_{2}-$ Abgabe (in $\mathrm{mm}^{3} \mathrm{Stdn}^{-1} \mathrm{~g}^{-1}$ ), obere Kurve $(\cdots \Delta \cdots) R Q$ in Abhängigkeit von der Dauer einer $2^{\circ}$-Behandlung. Ende der "lag period“ schraffiert. Zur Definition der "scores" vgl. Gre gory und Purvis 39 .

Abb. 5b. Wie Abb. 5 a, jedoch ganze Caryopsen des Petkuser Winterroggens (keine „lag period“).

nen - und ganz besonders die der WinterroggenEmbryonen - stark an, während sich der $R Q$ dem Wert 0,9 annähert. Von dem Zeitpunkt an, von dem ab eine Vernalisations-Behandlung an isolierten Embryonen nach P u rvis feststellbar wird, läßt die Atmungsintensität rasch nach, und der $R Q$ sinkt auf etwa 0,75 (vgl. Abb. 5).

39 F. G. Gregory u. G. N. P u rvis, Ann. Botany, N.S. 2, 237-251 [1938]. 
Zur Erklärung der „lag period“ nimmt Purvis an, daß in ganzen Caryopsen ein für die Vernalisations-Wirkung wichtiger Stoff (bei hohen wie bei tiefen Temperaturen) aus dem Endosperm in den Embryo übertrete, der in isolierten Embryonen erst neu gebildet werden müsse, wozu Kälte ebenso erforderlich sei wie für die Anhäufung dieses Stoffes in den Embryonen ganzer Caryopsen. Der natürliche Kontakt mit dem Endosperm konnte jedoch in P u rv is Versuchen nicht durch die Zugabe von Endosperm zum Medium ersetzt werden.

Auf Grund unserer Befunde erscheint es nun möglich, daß in der Zeit der überwiegenden Kohlenhydrat-Atmung, also innerhalb der ersten 14 Tage nach Vernalisations-Beginn, entweder (1) ein für die Vernalisations-Wirkung erforderlicher Stoff gebildet oder (2) aus einer Bindung oder inaktiven Vorstufe freigesetzt bzw. aktiviert oder (3) eine vernalisationshemmende Substanz zerstört oder inaktiviert wird. Die Begünstigung des Vernalisations-Effektes bei isolierten Embryonen durch Glucose-Zugabe läßt sich vielleicht damit begründen, daß die Glucose in der Zeit der Überführung einer thermolabilen „Vernalin"-Vorstufe in eine stabile der Erhaltungsatmung dient, der sonst die (den Annahmen 1 und 2 zufolge gebildete) labile „Vernalin“-Vorstufe zum Opfer fallen würde.

Zugunsten dieser letzten Annahme spricht auch ein Befund von Purvis ${ }^{11}$ : Bei einer Gesamt-Vernalisations-Dauer von 8 Wochen ist der VernalisationsEffekt, zumal nach „residual growth“-Behandlung, d.h. nach 4-tagelanger Veratmung der in isolierten Embryonen enthaltenen Kohlenhydrate bei $20^{\circ} \mathrm{C}$, größer, wenn in den ersten Vernalisations-Wochen (in denen vermutlich die Stabilisierung der labilen Vorstufe erfolgt) Zucker geboten wird und später nicht, als bei anfangs zuckerlosem Substrat und späterer Zuckerzugabe. - Auf alle Fälle wird es zur Bestätigung dieser Annahmen über den Zusammenhang zwischen der 14-tägigen Phase überwiegender Kohlenhydratatmung isolierter Embryonen einerseits und der ebenso langen Purvis schen "lag period“ andererseits noch weiterer Untersuchungen bedürfen.

Herrn Prof. Dr. G. Melchers danke ich auch an dieser Stelle vielmals für die Anregung zu den vorliegenden Untersuchungen, für die freundliche Aufnahme in seinem Institut sowie für zahlreiche förderliche Diskussionen. Der Deutschen Forschungsgemeins chaf $t$ bin ich für die finanzielle Unterstützung meiner Arbeiten zu Dank verbunden.

\title{
Beeinflussung der Wuchsformen des Lactobacillus bifidus
}

\author{
Von F. Petuely und F. Eichler \\ Aus dem Med.-chemischen Institut der Universität Graz \\ (Z. Naturforschg. 9 b, 229 234 [1954]; eingegangen am 29. September 1953)
}

\begin{abstract}
In der vorstehenden Arbeit wird gezeigt, daß der L. bifidus, der im Stuhl des an der Brust ernährten Säuglings praktisch ausschließlich als Stäbchen wächst, in vitro nur dann Verzweigungen verschiedenen Grades bildet, wenn die Nährböden bestimmte Hemmstoffe enthalten, bzw. die Nährböden nicht optimale Verhältnisse schaffen. Als ein solcher Stoff wurde der Bakterienhemmstoff aus dem sterilen Kulturfiltrat der Hefe Candida pseudotropicalis Basgal erkannt. Weiter wird nachgewiesen, daß auch Sulfathiazol und Sublimat in geringem Grade Verzweigungen bewirken. Kurz dauernde Einwirkung von Formaldehyd verursacht bei manchen Bifidus-Stämmen stärkste Verzweigungen. Alle Verzweigungen verschwinden wieder im Laufe einiger Passagen. Sterile Kulturfiltrate eines Stammes von E. coli und St.lactis verhinderten das Angehen von Bifidus-Kulturen, hemmten aber wachsende Kulturen nicht und bewirkten auch keine Verzweigungen. Zuletzt wird darauf hingewiesen, wie unwahrscheinlich es ist, daß Stämme von L. bifidus mit einer so großen Mutationsrate behaftet sind, daß sie nahezu konstant zu L. acidophilus mutieren, wie dies die erwähnten amerikanischen Autoren behaupten.

Die normale Wuchsform des L. bifidus ist die Stäbchenform.
\end{abstract}

$\mathrm{D}_{\mathrm{b}}^{\mathrm{i}}$ Morphologie und Physiologie des Lactobacillus bifidus bildete den Gegenstand zahlreicher Arbeiten verschiedener Autoren (Zusammen-

1 Ergebn. Hyg., Bakteriol., Immunitätsforsch. exp. Therap. 26, 193 [1949]. fassung siehe Boventer) ${ }^{1}$. Auch in neuester Zeit wurde dieses Thema von amerikanischen Autoren eingehend bearbeitet ${ }^{2}$. Die gleiche Forschergruppe

2 T o mar elli u. Mitarbb., J. biol. Chemistry 179, 485 [1949]; 181, 879 [1949]; 187, 197 [1950]. 